\title{
Clinical trial on solid 2nd generation platelet-concentrates in the management of the chronic osteomyelitis: A advanced regenerative surgeries.
}

\author{
Alessandro Crisci ${ }^{1,2,3, *} \mathrm{D}^{\prime}$ Adamo Raffaele ${ }^{2}$ and Michela Crisci ${ }^{4}$ \\ ${ }^{1}$ School of Medicine, University of Salerno Italy, 84084 Fisciano, SA, Italy; E-mail: alcrisci@unisa.it \\ 2 Unit of Dermosurgery Cutaneous Transplantations and Hard-to-Heal Wound, "Villa Fiorita" Private Hospital, 81031 Aversa, CE, \\ Italy; E-mail: raffaeledadamo@tiscali.it \\ ${ }^{3}$ Institute for the Studies and Care of Diabetics, Abetaia, 81020 Casagiove, CE, Italy; \\ ${ }^{4}$ Faculty of Medicine and Surgery, Vasile GoldisWestern University of Arad, 310025 Arad, Romania; \\ E-mail: criscimichela96@gmail.com
}

ORCID: https://orcid.org/0000-0002-4478-5714; https://orcid.org/0000-0002-1264-8467;

*Corresponding author: Prof. Alessandro Crisci, Department of Medicine, Surgery and Dentistry "Salernitan Medical School", University of Salerno, Fisciano (SA), Italy; E-mail: alcrisci@unisa.it; alessandrocrisci@libero.it; Phone: +39-3388722799

Research Registration Unique Identifying Number (UIN): Researchregistry: 5927;

https://www.researchregistry.com/browse-the-registry\#home/registrationdetails/5f3ce327bcff2b0018af5dde/

\begin{abstract}
The supposition is that the usage of fibrin rich in leukocytes and platelets advanced (A-PRF) in ulcerative osteomyelitis of the diabetic foot allows rehabilitation from this critical illness. In this investigation, the focus was to normalize the use of PRF in patients with osteomyelitis not amputated, to use this second generation platelet concentrate as a regeneration enabler. The researchers submitted and utilized A-PRF membranes (1300 g × $8 \mathrm{~min})$ in 7 patients (all diabetics) with osteomyelitis and cutaneous injury for 6 months. The membranes, in combination with the supernatant fluid produced by stress, have been integrated into the skin lesion down to the bone after surgical debridement. The advancement of the lesions after some period of time has been analyzed. All seven subjects had a Probe-to-Bone positive assay; MRI indicated a cortico-periosteal coagulation and/or foci of cortico-spongeous osteolysis contiguous to the lesion. Gram-positive bacterium were identified in our procedures in $52 \%$ of cases. Gram+ Cocci, for example, S. Aureus (15.6\%), S. Hemolytic (12.1\%), S. Viridans (7.1\%), and Gram-negative Bacteria, for example, Pseudomonas (10.6\%), Proteus (7.8\%), Enterobacter (5.7\%) are present. Candida Albicans is active in $2.8 \%$. The blood count shows no relevant differences.
\end{abstract}

To date, cutaneous lesions have cured in 6 of the seven subjects treated (one patient for more than five years) without any evidence of infection or recurrence. The results obtained on our subjects indicate that PRF membranes may be a therapeutic option in this problematic disease.

Keywords: cronic osteomyelitis; buffy coat; growth factor content; platelet-rich fibrin; concentrates of thrombocytes.

\section{Introduction}

Choukroun's Platelet Rich Fibrin (PRF) [1] is a 2nd generation platelet concentrates, substantially a overcoming of Platelet Rich Plasma (PRP), is, by these lines, another step forward in the therapeutic idea of platelet gel with a simplification formulation and small fake biochemical adjustments. Differently from other platelet concentrates, this procedure does not necessitate anticoagulants, thrombin, or some other gelificant, which makes the blood close to a natural centrifuge containing no additives. PRF can be created by simply activating the naturally occurring coagulation pathway without the assistance of anticoagulants or clotting promoters [2]. Although leukocyte platelets and cytokines play a relevant role in the biology of this biological material, the support matrix of fibrin is undoubtedly the unmistakable factor of the strong therapeutic capacity of L-PRF. Within a short the exclusion of an anticoagulant allows the activation of the vast majority of platelets included in the example to start the coagulation sequence. 
The osteomyelitis (OM) expressly alludes to bone marrow disease as contrasted with an osteitis in which the periosteum or cortical surface becomes contaminated through a penetrative lesion or wound. Regardless of these differentiations, the two are medically analyzed or treated in a corresponding way. Much has been reported on the diagnosis of $\mathrm{OM}$ in the long term and, even more critically, on how it attaches to diabetic foot ulcers (DFU). OM involving the diabetic foot frequently results from a collateral lesion or ulcer of the foot [3].

The global impact is from $1: 1,000$ to $1: 20,000$ people in Italy 19,000 cases/year, in Europe 100,000 cases/year. Male: The proportion of women is $2: 1$.

Bone and articulation infections are problematic for patients and humiliating for them and the medical service operators who treat them. The high success rates of antibiotic management in many infectious pathologies have not yet been realized in this condition. The variety of OM types necessitate distinctive clinical and surgical therapeutic methods. The different types of this syndrome incorporate, in a decreasing order of incidence: OM auxiliary to a adjacent concentration of contamination (after injury, surgery, or addition of a joint prosthesis) that is subordinate to vascular deficiency (in diabetic foot infections); finally the OM of the haematogenic origin. Chronic OM is associated with avascular bone necrosis and seizure augmentation (dead bone) and the surgical procedures are important for therapy despite antibiotic management. Contrary to what might be expected, acute OM can only respond to antibiotics. All in all, a multidisciplinary treatment approach is desirable for a successful outcome, including the abilities of muscle surgery, infectious diseases and plastic surgery, as well as vascular surgery, especially for complex presentations with delicate tissue disasters $[4,5]$.

The use of 2nd generation platelet concentrates in DFU with OM was not known to the authors until recently and was accepted by them unexpectedly (2018) [6]. This research describes the results obtained on seven subjects with chronic DFU osteomyelitis of the lower extremities (DFO).

\section{Material and methods}

\subsection{Preparing the PRF}

Blood was collected with the informed approval of each of the seven volunteers screened. All the contribution of the participants in this project were performed according to the ethical principles of the Institutional and Public Research Advisory Group and the 1964 Helsinki Declaration and its subsequent revisions. The Ethics Committee has waived an ethical order for this survey because blood was not used as a recognizable resource [7] (Research Registry: No. 5927).

Factors affecting fibrin coagulation growth and structure incorporate hereditary components, stimulating factors, (e.g., irregular convergence of thrombin and factor XIII in plasma, blood flow, platelet activation, oxidative stress, hyperglycemia, hyperhomocysteinemia, drugs and tobacco smoke) and various parameters (e.g., microgravity, $\mathrm{pH}$, temperature) $[7,8]$. It has been stated that all donors are carriers of chronic osteomyelitis from diabetic lower limb ulcers.

The blood count of donors was also examined before starting the studies to determine the standard blood cell enumeration interval. In order to quantitatively describe and follow the clinical sequence of reparative, a severity rating of the lesion was set by examining the lesion and marking the various clinical and anatomical patient factors (Wound Severity Score Tabb.1, 2, 3 and 4). The blood was then collected in A-PRF glass tubes (Advanced PRF) without anti-coagulant or gel separator ( $9.0 \mathrm{ml} \mathrm{A-PRF} \mathrm{Serum} \mathrm{Vacutainer)} \mathrm{to} \mathrm{create} \mathrm{clots} \mathrm{and} \mathrm{PRF} \mathrm{membranes.} \mathrm{The} \mathrm{blood} \mathrm{was}$ immediately collected with a needle in tubes (average value 22", less than 25" per tube) and instantly (within 1 minute) centrifuged from the representation at a temperature above $21^{\circ} \mathrm{C}$ (somewhere in the range of $21^{\circ}$ and $30^{\circ} \mathrm{C}$ ). Using the L-PRF Wound Box, the membrane stress procedure in coagulation is performed through light, homogeneous stress, and the resulting membrane remains consistently moist and evenly wet in serum. The PRF fabrication method is extremely simple and requires only a blood test and a DUO Quattro per PRF tabletop centrifuge specifically designed for this method (DUO Quattro per PRF) [6-9]. 
The subsequent protocol is as described below: blood samples are collected in $9 \mathrm{~mL}$ glass tubes, without anticoagulant or splitting gel, and are rapidly spinned according to the recommended time interval: 30 sec acceleration, 8 min at $1300 \mathrm{rpm}$ (189 g), $36 \mathrm{sec}$ deceleration and stop. After spin drying, three sections are positioned in the tube: the red cells at the bottom, the fibrin coagulation that corresponds to the PRF in the center and the acellular plasma at the top. The fibrin clotting is separated from the tube with sterile clips and the PRF is obtained by removing the red cloth from its lower end. The realization of this method is completely based on the ease of blood collection and the speed of movement in the centrifuge [10].

The entire procedure should be carried out consistently in a sterile manner, based on the fact that the growth factors contained stimulate tissue regeneration and, therefore, presumably also cell regeneration. This delicate technique preserves the extraction and acute significant amount of growth factors. The available PRF-Boxes will be accessible in a set of shapes and will exercise, through the stress plate, various exercises depending on the weight, offering to climb to a membrane of varying density, variable width and length. The Wound L-PRF Box is planned by AA. [10] It consists of a $17.5 \times 7.6 \times 2 \mathrm{~cm}$ metal container containing a perforated steel plate measuring $150 \times 68 \times 1.5 \mathrm{~mm}$. There is a second steel plate that acts as a compressor, measuring $150 \times 68 \times 1.5 \mathrm{~mm}$, weighing 148 grams. This second type of $\mathrm{U}$-shaped plate uses a mass of $142.437 \mathrm{~Pa} / \mathrm{cm}^{2}$. In this investigation, the stress to create the membranes was applied to the clot for 2 minutes. Each membrane is separated into three equivalent size areas: proximal (head), central (body) and distal (tail) through a sterile scalpel cut. Only the proximal portion of the membrane has been used $[9,10]$ and the central portion only if important.

With the PRF method, blood coagulation occurs immediately after sampling, directly after contact with the glass surface of the tube, due to the elimination of anticoagulation. In the remote possibility that the time required for blood collection and the start of centrifugation (several revolutions per minute, $\mathrm{g} / \mathrm{min}$ ) is very retarded, the polymerization of the fibrin is of such a magnitude that only a small aspect of the coagulation that is not consistent (PRF-like) will be obtained. Next, blood acquisition must be quick and easy, driven by rapid centrifugation, and is essential in the PRF yield specification. It is defined to provide a thickness of approximately $3 \mathrm{~mm}(3.08 \pm 0.5)[10]$, an evenly hydrated membrane with an exudate rich in platelets, leukocytes, vitronectin and fibronectin, expressed in the structure of the fibrin network with CD34+ hematopoietic stem cells. [11].

The fibrinogen is originally focused in the upper part of the tube until it causes the creation of the autologous circulating thrombin that transforms it into the fibrin mesh. The result is the coagulation of fibrin containing platelets located in the middle, directly between the lower membrane of the red platelets and the upper acellular part of the plasma. The PRF coagulation thus acquired is then placed on the bottom of the L-PRF Box and crushed with the compressor cover. This approach forms an autologous fibrin membrane. The L-PRF box is intended for the creation of constant thickness membranes that remain hydrated for a few hours and allow the recovery of serum exudate expressed by fibrin coagulation, this is rich in proteins, such as vitronectin and fibronectin [7]. The L-PRF clot has all the potential to be responsible for a slow release of growth agents and glycoproteins from the matrix ( $\geq 7 \mathrm{dd}$, up to 28 dd) [8]. Adherent proteins: fibrinogen (Fg), fibronectin (Fn), vitronectin (Vn) and thrombospondin-1 (TSP-1) are copious in the fibrin structure. The growth factors accumulated in the platelets and which are essential for wound regeneration include PDGF, with - $A B$ and - C; they are also present as VEGF-A, TGF-1, EGF (Fig. 1), FGF-2, HGF, and growth factor similar to insulin-1 (IGF-1). 

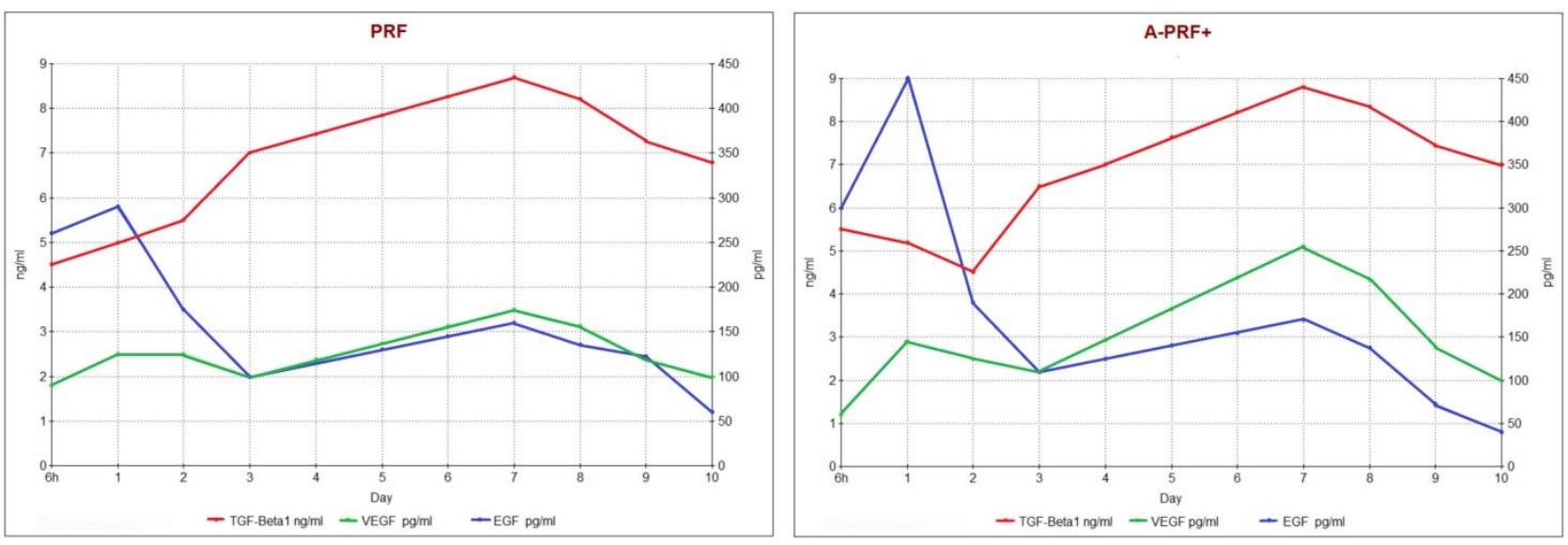

Figure 1. Several concentrations of growth agents TGF- $\beta 1$, VEGF, EGF over time generated by L-PRF and A-PRF. Statistical analysis of growth factor emissions by time points as mean \pm standard deviation for PRF and A-PRF+. VEGF, TGF- $\beta 1$ release, EGF release.

Analyzing three pro-inflammatory cytokines (IL-1 $\beta$, IL-6, TNF- $\alpha$ ), one inflammatory cytokine (IL-4) and one angiogenesis activator (VEGF), it was suggested that PRF might also be a focus of immune modeling with the potential to control inflammation and multiplication of non-differentiated adult cells, comprising CD34+ precursor cells, MSCs (mesenchymal stem cells), SMC progenitors (smooth muscle cells) and endothelial precursors [8-11]. The multiple power of these types of stem cells and their increased capacity to increase vascular tissue repair and through paracrine mechanisms also make them as therapeutic vehicles in restorative medicine. In addition, tissue damage produces severe chemo-attractive signals for stem cells, creating the precondition for their restorative activity. Platelets control the recruitment of adult stem cells to damaged cells and can, in this way, be a considerable tool in the execution of restorative cell reactions. Activated platelets discharge HGF and have been depicted to advance MSC absorption in human endothelial cells. Human stem cell proliferation (hMSCs) is related to platelet count in A-PRF.

\subsection{Blood chemical tests}

Blood samples of each patient were also taken to perform a complete blood count (CBC) using K3E tubes with $5.4 \mathrm{mg}$ EDTA (VacuMed). As reported by previous investigation [6-11], three blood samples were taken from the left brachial vein of each person through an 18-gauge needle, two for PRF generation and one for cellular blood count. The samples were processed with a Cell Dyn $3500 \mathrm{R}$ cell counter (ABBOTT). The predictive evaluation of OM in the considered subjects was delivered by Probe-to-Bone (PTB) technique and then by MRI and bone cell culture for bacterial testing.

\subsection{A-PRF grafting protocol}

Each of the seven patients, after adequate preparation (suspension of anticoagulant drugs for at least 7 days and low molecular weight subcutaneous heparin) underwent surgical debridement, under subarachnoid sedation, in the operating room, with the evacuation of imperative and conceivable bone portions in the lower part of the wound, including the execution of the planned bacterial cultivations tests. Extremity vasodilator drugs (Iloprost, Alprostadil) have not been used. This study population excluded patients with a complicated hospital stay who stayed in the intensive care unit stay. After cleaning the surgical wound with a half combination of hydrogen peroxide and iodopovidone and proper control of hemostasis by electrocautery, A-PRF was transformed into membranes after coagulation stress for 2 minutes. The supernatant obtained from the crushing was taken from the Wound L-PRF Box with a sterile 10cc syringe and was completely incorporated into the lesion in depth together with the PRF fragment that includes the proximal third of the A-PRF membrane [9 - 11] which acts as a biological glue through which cells can migrate. Before the insertion of PRF the wound was washed with hydrogen peroxide, as the active drainage inhibits the activity of growth factors. The dressing was performed with oily gauze, sterile gauze, germanic cotton and flexible cement cloth. The post-surgical drug therapy was with levofloxacin $500 \mathrm{mg} \mathrm{cp}, 1 \mathrm{cp}$ per day for 5 days, and low atomic weight heparin (enoxaparin sodium) for 7 days, though the drugs that each subject constantly takes for several 
pathologies have been hired. Based on lifestyle and antibiogram consequences, specific antibiotics for generic use for 15 days have been included. The main drugs were administered for 7 days. Subjects were inspected every week in the ambulatory until they healed. In the remote possibility that there was no indication of wound recovery, the PRF was reapplied 5 weeks after the first time. All accumulations of PRF were removed with water and sterile cloth at the first dressing. Patients proceeded with the refreshment dressing routine between PRF treatments, as it had already been used. Two subjects had to perform the surgical procedure a second time after 40 days. No direct patient identifiers were maintained in the study database.

\subsection{Wound Gravity Index Score}

The severity score of the lesion was calculated according to clinical and anatomical conditions and by considering the lesion and the patient's factors. The points were awarded in a self-assertive and considered manner using the usual clinical experience on wound repair. These overall parameters of the lesion are recorded in Table 2. Anatomical contemplations have been recorded and obtained, for example, the presence of uncovered bone or a wound area of the ligament, and the nature of the pulsation of the pedidial artery and posterior tibial (and the area compared with the wound area) (Table 3). The lesions have been estimated to decide the absolute territory of the lesion, the depth and the degree of openness. The estimation of the lesion surface was dictated by photographing the lesion and comparing it with a strip graded to the millimeter and then analyzed with an estimation software (measurement IC 2.0.0.133), discovered free of charge on the web.

Three estimates were made and the average surface area was the normal of the three valuations. The extent of the lesion was controlled by the set of measurements on the patient. The scores attributed to these different assessments of the lesion can be found in Table 4. Initial and subsequent wound scores were recorded and organized at each visit to each facility by two clinical agents and medical personnel in charge of wound recovery. These conclusions were periodically counted by the examiner who proposed them.

The wound severity score is shown in Table 1 for each patient.

\subsection{Definition of successful therapy}

A lesion was called recovered when it was repaired with a new epithelium. This was externally resolved during the evaluation of the lesion performed during the next routine procedure. At each visit, estimates and photos of the lesion were taken to report progression. The result of the treatment is determined by the rate of variance of the area and volume of the surface, determined as the least estimation each day of the preliminary assessment separated from the baseline estimation (IDR) (Fig. 2).

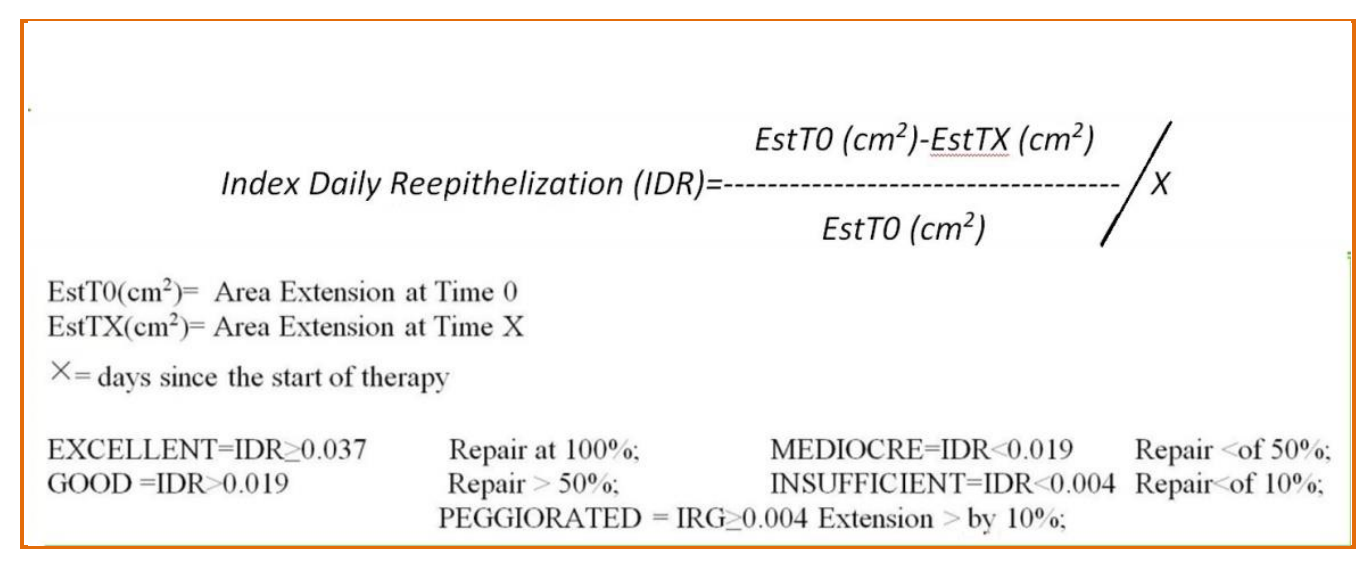

Figure 2. Daily reepithelialisation index (DRI).

\section{Results}

The Authors have produced and used A-PRF membranes based on venous blood, in patients suffering from notamputed osteomyelitis, with skin lesions that have been present, in any case, for a semester. The membranes, 
together with the fluid obtained from the stress of the Wound L-PRF Box, were inserted into the injured skin, deep down, after surgical debridement. The progress of the lesions has been studied after some time.

The results obtained with this method are shown in Table 1, together with the general features of the processed patients.

Table 1. Patient specifications

\begin{tabular}{|c|c|c|c|c|c|c|c|c|c|c|c|}
\hline $\begin{array}{c}\text { № } \\
\text { patient }\end{array}$ & $\begin{array}{l}\text { Aging } \\
\text { (years) }\end{array}$ & Gender & $\begin{array}{l}\text { Length of } \\
\text { Stay (days) }\end{array}$ & $\begin{array}{l}\text { Concomitant } \\
\text { Diseases }\end{array}$ & $\overline{D D Y}$ & $\begin{array}{l}\text { Wound size } \\
(\mathrm{cm}) \\
\mathrm{L} \times \mathrm{W} \times \mathrm{H}\end{array}$ & $\begin{array}{l}\text { Position of } \\
\text { the wound }\end{array}$ & $\begin{array}{l}\text { Therapy } \\
\text { period }\end{array}$ & $\begin{array}{l}\text { Follow- } \\
\text { up(days) }\end{array}$ & Result & $\begin{array}{c}\text { Total } \\
\text { Severity } \\
\text { Score }\end{array}$ \\
\hline 1 & 68 & Male & 103 & $\begin{array}{l}\text { PAD, } \\
\text { DFO }\end{array}$ & 25 & $2 \times 2 \times 5$ & $\begin{array}{c}\text { Lower third } \\
\text { left leg }\end{array}$ & 40 & 1825 & $\begin{array}{l}\text { Automatic } \\
\text { closing }\end{array}$ & 25 \\
\hline 2 & 71 & Female & 64 & $\begin{array}{c}\text { PAD, } \\
\text { DFO, HTN }\end{array}$ & 40 & $1 \times 1 \times 2$ & $\begin{array}{l}\text { Right } V^{\circ} \\
\text { finger foot }\end{array}$ & 25 & 365 & $\begin{array}{l}\text { Automatic } \\
\text { closing }\end{array}$ & 24 \\
\hline 3 & 63 & Female & 56 & $\begin{array}{c}\text { PAD, } \\
\text { DFO, ESRD }\end{array}$ & 24 & $2 \times 2 \times 2$ & Right Plantar & 32 & 180 & $\begin{array}{c}\text { Automatic } \\
\text { closing, } \\
\text { deceased for } \\
\text { CAD } \\
\end{array}$ & 40 \\
\hline 4 & 60 & Male & 46 & $\begin{array}{c}\text { PAD, } \\
\text { DFO, HTN }\end{array}$ & 20 & $1 \times 3 \times 5$ & $\begin{array}{l}\text { Left } V^{\circ} \\
\text { finger foot }\end{array}$ & 27 & 120 & $\begin{array}{l}\text { Automatic } \\
\text { closing }\end{array}$ & 15 \\
\hline 5 & 66 & Male & 45 & $\begin{array}{c}\text { PAD, } \\
\text { DFO, ESRD }\end{array}$ & 15 & $1 \times 2 \times 5$ & $\begin{array}{c}\text { Right } \mathrm{I}^{\circ} \\
\text { finger foot }\end{array}$ & 33 & 211 & $\begin{array}{l}\text { Automatic } \\
\text { closing }\end{array}$ & 20 \\
\hline 6 & 58 & Male & 94 & $\begin{array}{c}\text { PAD, } \\
\text { DFO, HTN }\end{array}$ & 23 & $1 \times 1 \times 5$ & $\begin{array}{c}\text { Left toe } \\
\text { plantar }\end{array}$ & 33 & 388 & $\begin{array}{l}\text { Automatic } \\
\text { closing }\end{array}$ & 35 \\
\hline 7 & 70 & Male & 86 & $\begin{array}{c}\text { PAD, } \\
\text { DFO, HTN }\end{array}$ & 35 & $2 \times 2 \times 5$ & $\begin{array}{l}\text { Right toe } \\
\text { plantar }\end{array}$ & 30 & 905 & $\begin{array}{l}\text { Automatic } \\
\text { closing }\end{array}$ & 23 \\
\hline $\begin{array}{l}\text { Average } \\
\pm \text { D.S. }\end{array}$ & $\begin{array}{l}65.14 \\
\pm 4.61 \\
\end{array}$ & $5: 2$ & $\begin{array}{r}70.57 \\
\pm 22.88 \\
\end{array}$ & & $\begin{array}{r}26.0 \\
\pm 8.0 \\
\end{array}$ & $1.4 \times 1.8 \times 4.1$ & & $\begin{array}{l}31.43 \\
\pm 4.5 \\
\end{array}$ & $\begin{array}{c}570.6 \\
\pm 566.4 \\
\end{array}$ & & $\begin{array}{r}26.0 \\
\pm 8.0 \\
\end{array}$ \\
\hline
\end{tabular}

*the improvement is defined as the regeneration of the limb wound within a year and a half, but without the wound closure. DDY: year of diagnosis of diabetes; PAD: peripheral arteriopathy; ESRD: end-stage renal disease; HTN - arterial hypertension; CAD: Coronary Artery Disease; DFO: Diabetic Foot Osteomyelitis;

Table 2. Complete the general criteria of the injury score.

\begin{tabular}{|l|c|c|c|c|}
\hline & None & Mild & Marked & Complete \\
\hline Periwound Erythema & 0 & 2 & 5 & 7 \\
\hline Periwound Oedema & 1 & 2 & 4 & 7 \\
\hline Injury purulence & 0 & 3 & 4 & 7 \\
\hline Injury fibrin & 0 & 2 & 5 & 7 \\
\hline Limb pitting Oedema & 1 & 2 & 4 & 7 \\
\hline Limb brawny Oedema & 0 & 3 & 4 & 0 \\
\hline Injury granulation & 0 & 0 & 0 & \\
\hline
\end{tabular}

Table 3. Anatomical wound score considerations.

\begin{tabular}{|c|c|c|c|c|c|c|c|}
\hline \multicolumn{4}{|c|}{} & \multicolumn{2}{c|}{ Dorsalis } & \multicolumn{2}{c|}{ Posterior } \\
\hline Visible bone & Score & Visible Tendon & Score & Pedis Pulse & Score & Tibial Pulse & Score \\
\hline Yes & 10 & Yes & 7 & $0-1+$ & 5 & $0-1+$ & 5 \\
\hline no & 0 & No & 0 & $2+$ & 2 & $2+$ & 2 \\
\hline \multicolumn{2}{|r|}{} & & $3-4+$ & 0 & $3-4+$ & 0 \\
\hline
\end{tabular}

Table 4. Wound Score-Wound Measurements.

\begin{tabular}{|c|c|c|c|c|c|c|c|}
\hline $\begin{array}{c}\text { Measure } \\
(\mathrm{cm} 2)\end{array}$ & Score & Width (mm) & Score & $\begin{array}{l}\text { Indefinite } \\
(\mathrm{mm})\end{array}$ & Score & Lifetime & Score \\
\hline$<1$ & 0 & $<5$ & 0 & $<2$ & 3 & $<8 \mathrm{wk}$ & 0 \\
\hline $1-2$ & 1 & $5-10$ & 3 & $2-5$ & 5 & $8 \mathrm{wk}-6 \mathrm{mo}$ & 1 \\
\hline $2-5$ & 3 & $10-20$ & 7 & $>5$ & 8 & $6 \mathrm{mo}-1 \mathrm{yr}$ & 2 \\
\hline $5-10$ & 6 & $>20$ & 10 & & & $2-3 \mathrm{yr}$ & 5 \\
\hline $10-30$ & 8 & & & & & $5-10 \mathrm{yr}$ & 7 \\
\hline$>30$ & 10 & & & & & $10 \mathrm{yr}$ & 9 \\
\hline
\end{tabular}


The average age of patients processed is 65.14 years \pm 4.61 , with a male/female relationship of $5 / 2$, all subjects were affected by Chronic Osteomyelitis and Chronic Obstructive Arteriopathy in non-insulin-subordinate Diabetic Disease examined for 26.00 years \pm 8.0 in the norm. The average duration of osteomyelitis was $70.57 \pm 22.88$ days. The standard score of absolute severity of treated subjects was $26.0 \pm 8.0$ (Tab.1).

All patients indicated positivity in the Probe-to-Bone test, and the Nuclear MRI showed cortico-periostal necrosis or potentially osteolysis with cortico-elastic, adjacent to the lesion. Osteonecrosis was also present with bone fracture and dissolution (Figs. 3-5).

Gram-positive microorganisms were found in our subjects in 52\% of cases. Other discovered germs include Grampositive cocci, for example, S. Aureus (15.6\%), $\beta$-hemolytic Streptococci (12.1\%), S. Viridans (7.1\%) and Gram-negative bacillus, for example, Pseudomonas (10,6\%), Proteus (7.8\%), Enterobacter (5.7\%). Candida was present in $2.8 \%$ of cases.

The normal terapy time after PRF grafting was $31.43 \pm 4.5$ days. The average growth to date was $570.6 \pm 566.4$ days.

\section{Discussion}

A key limitation of this study was the small sample size of the DFO population studied ( $N=7)$, limited by the number of DFO patients that can undergo regenerative surgery available within the hospital.
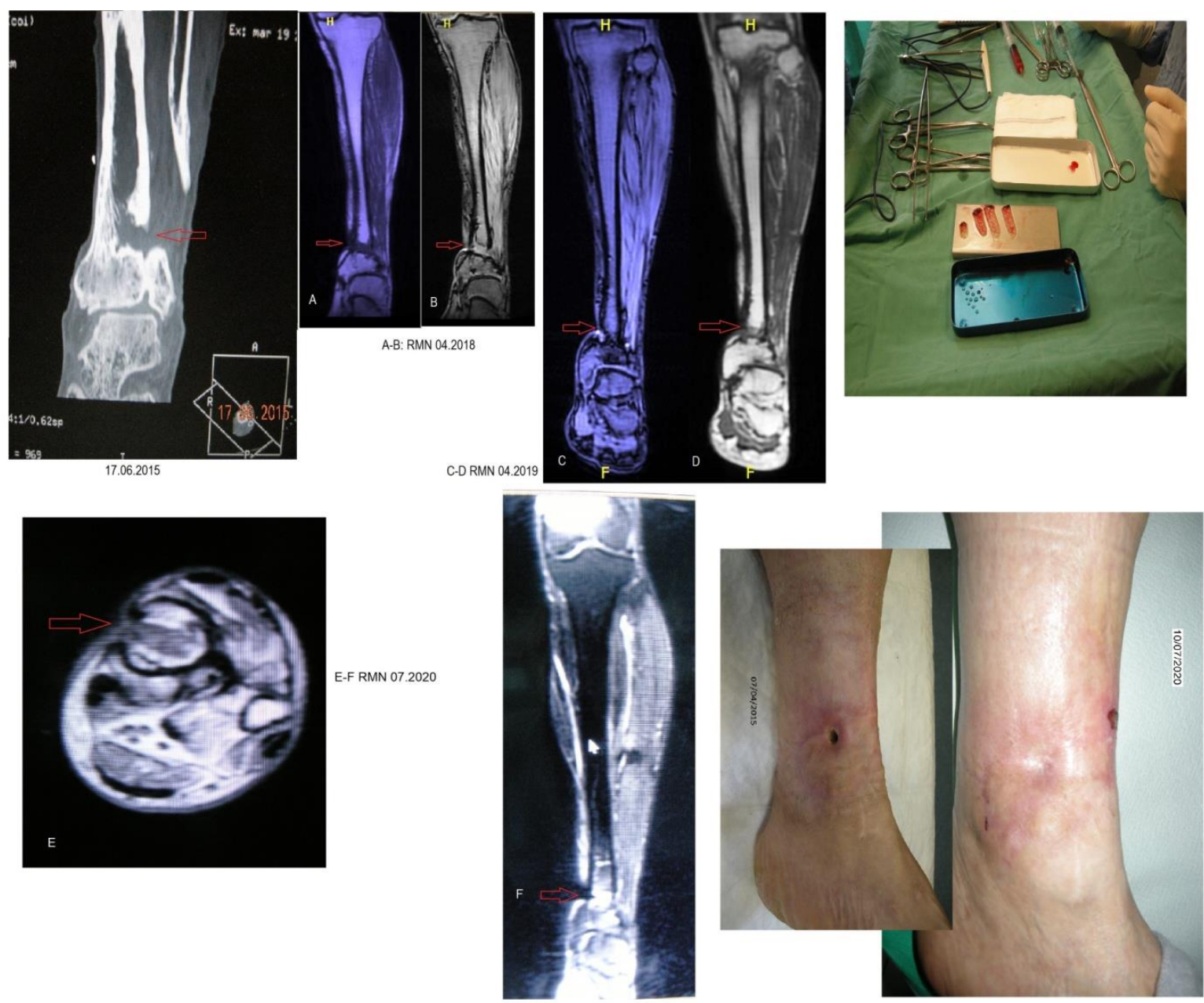

Figure 3. Patient $\mathrm{N}^{\circ} 1$. In A-C-D-E-F, MRI at various stages of wound development until healing, stable after more than five years. Some skeletal regrowth is also appreciated at the recent MRI (from Crisci et al. 2020)[22]. 

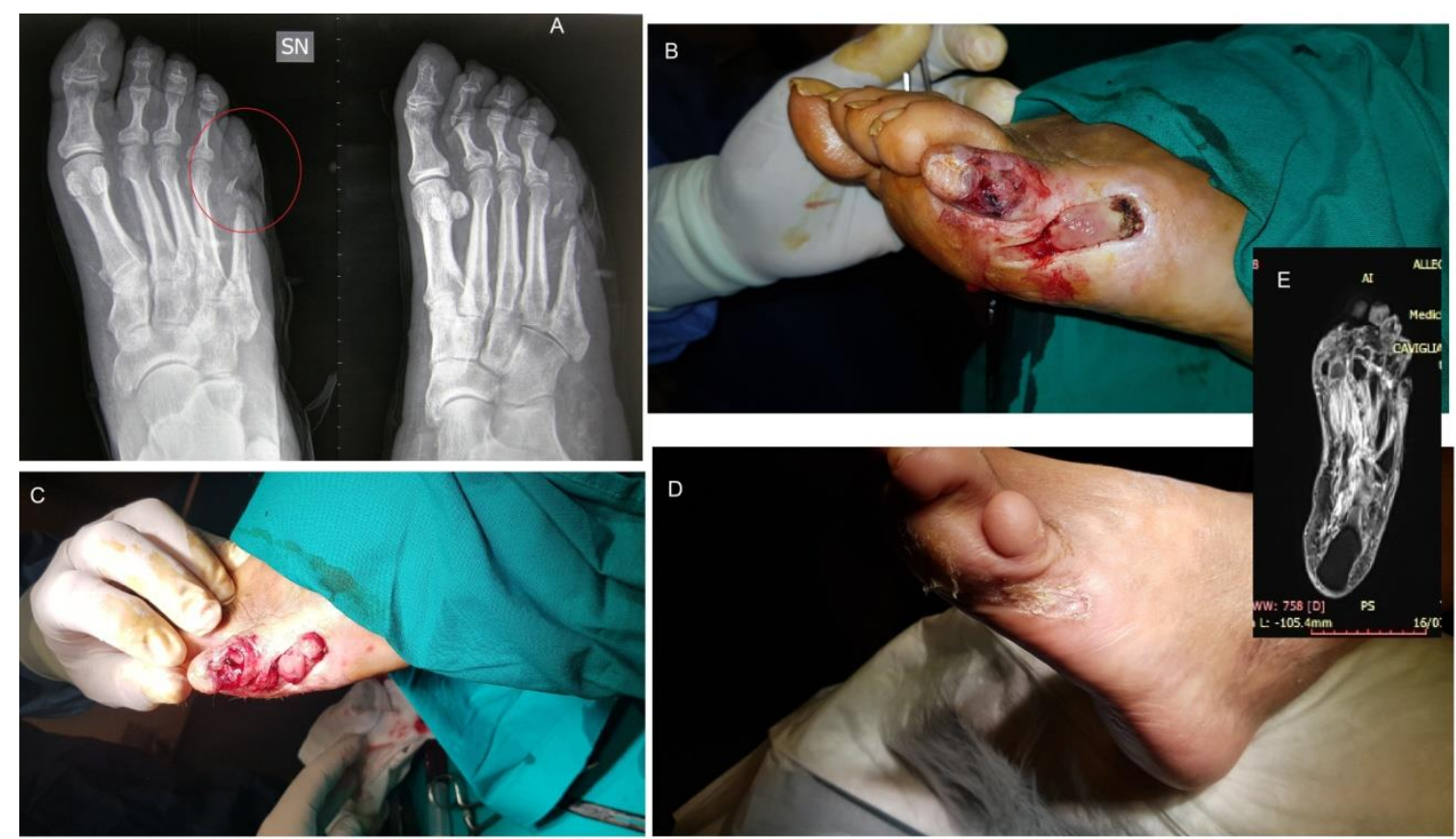

Figure 4. Patient $\mathrm{N}^{\circ} 4$. In a fifth digital X-ray imaging situation, B: intraoperative status; C: L-PRF graft deep in the wound; D: wound progression to injury healing after 4 months and only one PRF graft; E: RNM from 07.16.2020 once healing is obtained (from Crisci et al.2020)[22].
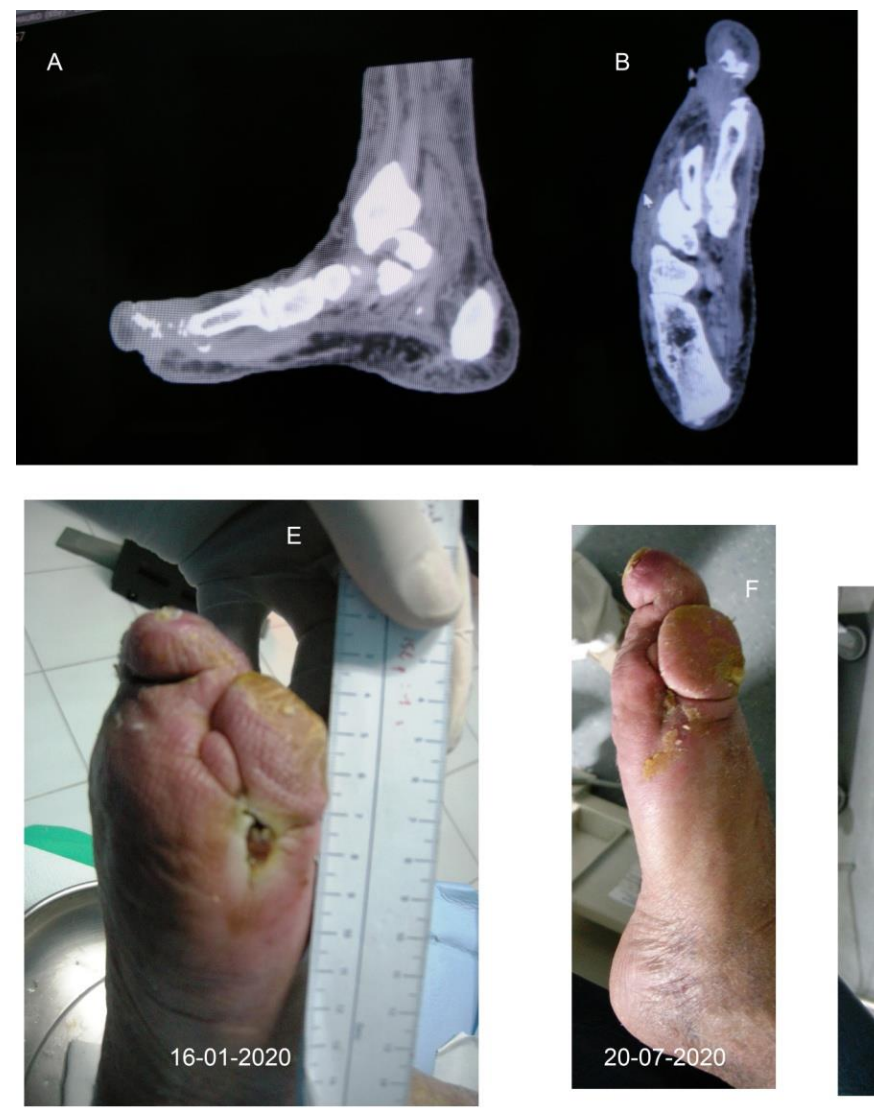
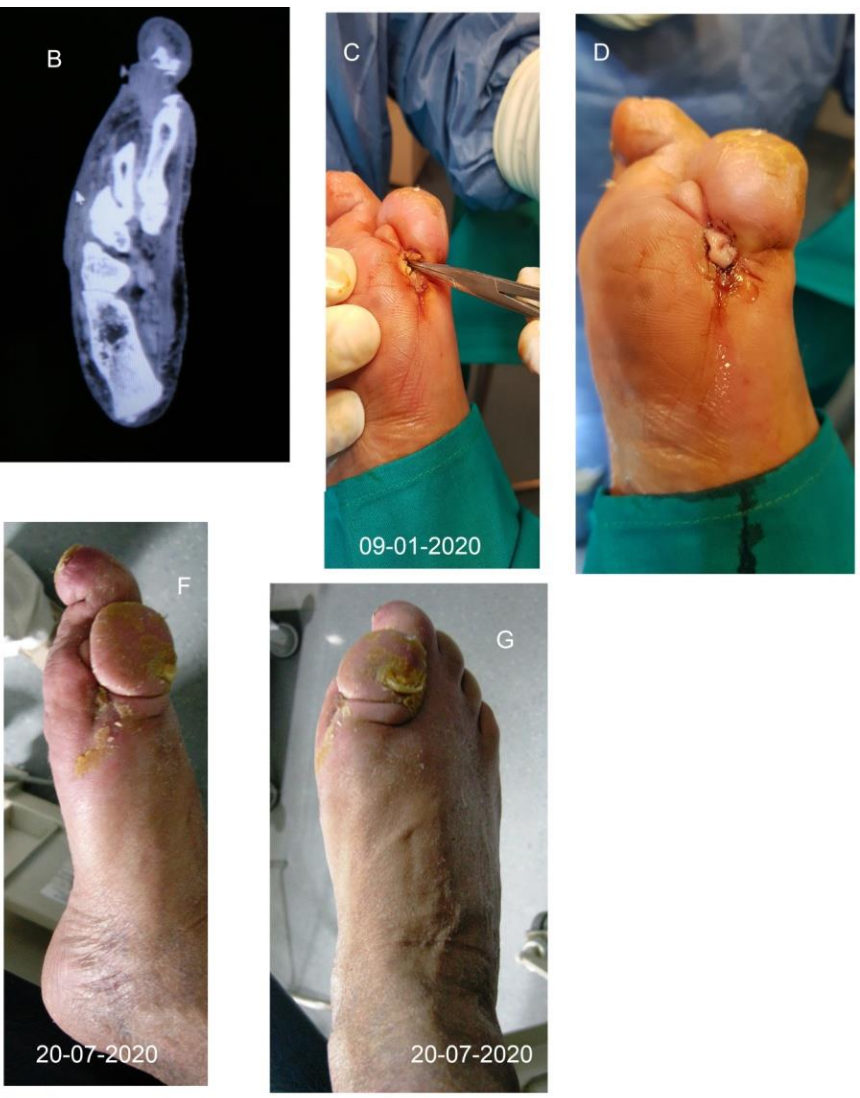

Figure 5. Patient $N^{\circ} 5$. In A and $B$ MRI status of 1st digit, C: intraoperative status; D: L: L-PRF graft deep in the wounds; E, F, G: injury progression until closure after 6 months and only one PRF graft (from Crisci et al.2020)[22].

Up to this point, skin osteomyelitis injuries have been recuperated in all treated subjects (only one subject died of cardiovascular causes two years after surgery, however, the injury was completely healed at that point), without any evidence of contamination or backslide. In one of the patients ( $\left.N^{\circ} 1\right)$ we are witnessing a specific bone 
regrowth more than five years after the closure of the skin lesion (Fig. 2F). The use of PRF in the treatment of skin lesions of the foot by the Authors has led to the announced results, with a moderate effort in terms of surgical strategy and monetary expenses for the medical office where patients were treated. In addition, the surgical hazard to which the patient has occurred is further low.

The treatment of DFU is tied to a huge budgetary burden, and the cost increases with the delay remain together with the need for surgery. The presence of osteomyelitis is a basic factor for significant expenses, the longer duration of the clinic remains $\overline{\bar{T}}_{\overline{\bar{T}}}$ with the long-range use of anti-infection agents and the need for removal. Moreover, of the apparent multitude of factors that influence the qualitive scar condition, what seems to have the best effect is the time needed to repair a lesion [13]. The extensive writing supports the case that the injury that recovers within 21 days limits abnormal healing. Secondly, one of the basic areas of research on consumption and recovery of wounds is to explain the pathophysiology of the wound correction measure, shortening the time of healing of the wound, the danger factors defined by the scarring procedure and the transformation of this information into therapeutic provisions. The use of PRF in compressed format as a speeding up wound repair agent seems to warrant its use. The integration of leukocytes into PRF should be carefully examined especially when the bio-material is used for injury repair and when scar formation is a significant preoccupation.

One aspect to consider is the inclusion of leukocytes. Marx's (1988) impression that the perfect platelet concentration rate was 3-4 times in the PRP provisions suggests the need to stay away from considerably present out platelets and the presence of leukocytes. Not long ago, this was the subject of discussion: some specialists, including the AA argue that leukocytes should be incorporated to encourage wound debridement, wound recovery and subsequent tissue regeneration, while some are concerned about the surprising intensification of the worsening [14]. In this way, additional researches and investigations should culminate in solutions.

In this research, each of the seven patients performed the "Probe-To-Bone Test" with positive results, MRI revealed cortico-periosteal colliquation and the central areas of cortico-spongeous osteolysis with reduced sign strength in the skin lesion region. Oedemas due to septic irritation and delicate tissue abscesses have also been discovered (Tab. 4). In our subjects, as often happens in chronic injury, some germs have been found at the same time: microorganisms are the most known cells, but contagious contamination has also been identified. Treatment of constant osteomyelitis currently includes surgical treatment, anti-infective treatment, hyperbaric oxygen treatment (OTI), active anti-bacterial stimulation (ITSB). Surgical therapy is the basic of treatment [16]. The focus is the removal of contamination and the practical reconstruction of the treated bone fragment. With the surgeries received so far for the treatment of osteomyelitis, the possibility of annihilating the disease is to eliminate the infected bone and all contiguous tissue down to the essential healthy tissue. Occasionally, however, a small evacuation may serve the purpose, which guarantees neither the stability nor the capacity of the treated limb, yet most of the time, after the removal of the infected bone, a rational surgical reconstruction is necessary. Together with the contaminated fabrics, it is equally appropriate to eliminate all internal fixation systems (plates, screws, nails, staples, etc.) present in the infected area, and fall back on another external adjustment. All modern surgical practices can offer amazing results, despite the length and order of the incredibly long medications and the not inconsiderable danger of complications and disappointments. The use of A-PRF in osteomyelitis lesions treated by us has given the announced results with a moderate involvement in the surgical method and financing of the healthcare facility where the patient is operated. In addition, the surgical danger to the patient is even lower (our patients have been fully treated under subarachnoid sedation).

Finally, the effect of PRF on bone cells should not be due to the effect of a solitary growth factor, but to the combined impact of several platelet growth factors.

Additional medical, histological, verifiable investigations are required to understand the benefit of this new procedure. Nevertheless, it cannot be ignored that, that is obtained from an autologous blood withdrawal, the delivered PRF is scarce and only a limited volume can be used. This situation limits the efficient use of PRF in huge osteomyelitis lesions. Although the possible uses of PRF are extensive, precise information on the functioning of the biomaterial, its science, expertise and cutting points is needed to improve its use in daily clinical application. 


\section{Conclusions}

In general, A-PRF is strong, suitable to bear weights, has a capacity of two overlaps to extend under stress, and keeps the surgical points so that at least two membranes can be surgically sewn (it essentially deforms before tearing) modulus: $0.2 \mathrm{MPa}$; strain: $140 \%$; breaking energy: $3.2 \mathrm{~N} . \mathrm{mm}$ )[16-20]. The membrane has demonstrated a fissure resistance comparable to the rupture of a perfectly flawless aorta and much higher than traditional PRP clots [21].

The use of A-PRF in cases of OM from a diabetic foot ulcer will increase our understanding of wound repair, in particular in the regenerative treatment of chronic skin lesions. The study aimed to standardize the use of PRF in patients with osteomyelitis, to use this second-generation solid platelet concentrate, increasing recovery capacity.

The results obtained in these seven cases suggest that PRF membranes may be a therapeutic option in this problematic pathology. Starting from this project, we intend to create a randomized trial to confirm the clinical impact of A-PRF and its substitutes, for example i-PRF, also as a component of its antibacterial action. The significant reasons for clarifying the conceivable modifiability that can be seen in the results could be attributed to the types of platelet concentrates used (PRP, PRF) that may change in structure (gel or fluid), as well as in platelet concentration, leukocyte content, network density. Fibrin in activation mode can normally occur by tissue contact or, can be stimulated by thrombin or calcium chloride.

The Authors suggest to use a product containing leukocytes and platelets in a mixture after debridement surgery to reduce the bacterial load (these eliminate cells and hinder the growth of biomembranes) and stimulate healing. Although second-generation platelet concentrates (PC) weakly influence tissue regeneration alone, these biomaterials may have the potential to increase the viability of an essential treatment or to initiate treatment, e.g., surgical or pharmacological, by observing an update of the insensitive reaction to antigens [14]. PC treatment can also be seen as a "substitution therapy".

In both cases, PCs give the important components for tissue recovery, including growth factors and structure materials, which cannot be provided justifiably by surgery or drugs.

The Authors believe that this work will be one of the bases for future investigations to further research the commitment of leukocytes in the PRF arrangement to achieve ideal planning both to fight diseases and to advance adequately wound healing, particularly in cases of chronic osteomyelitis (DFO).

Conflicts of interest: The authors do not represent any conflicts of interests.

Contributions of the author: Conceptualization, original drawing up and preparation of drafts, A.C.; Approval and data processing, D.R.; Methodology and Approval, M.C;

Funding: This study has not been specifically funded by public sector, commercial or non-profit funding agencies.

\section{References}

1. Choukroun, J.; Adda, F.; Schoeffler, C.; Vergelle, A.; Une opportunité en paro-implantologie: le PRF. Implantodontie, 2010, 62, 42-55.

2. Toffler, M.; Toscano, N.; Holtzclaw, D.; Del Corso, M.; Dohan Ehrenfest, D.; Introducing, Choukroun mins platelet rich fibrin (PRF) to reconstructive surgery milieu. J.I.A.C.D., 2009, 6, 21-32.

3. Lew, D.P.; Waldvogel, F.A.; Osteomyelitis. Lancet, 2004, 364, 369-379.

4. Calhoun, J.H.; Manring, M.M.; Shirtliff, M.; Osteomyelitis of the Long Bones. Seminars in Plastic Surgery, 2009, 23, 59-72. http://doi: 10.1055 / s-0029-1214158

5. Gogia, J.S.; Meehan, J.P.; Di Cesare, P.E.; Jamali, A.A.; Local antibiotic therapy in ostemyelitis. Seminars in Plastic Surgery, 2009, 23, 100-107. http://doi: 10.1055/s-0029-1214162 
6. Crisci, A.; Marotta, G.; Licito, A.; Serra, E.; Benincasa, G.; Crisci, M.; Use of leukocyte platelet (L-PRF) rich fibrin in diabetic foot ulcer with osteomyelitis (three clinical cases report), Diseases, 2018, 6, 30. http://doi:10.3390/diseases6020030.

7. Miron, R.J.; Fujioka-Kobayashi, M.; Hernandez, M.; Kandalam, U.; Zhang, Y.; Ghanaati, S.; Choukroun, J.; Injectable platelet rich fibrin (i-PRF): opportunities in regenerative dentistry? Clinical oral investigations, 2017, 21, 2619-2627. http://doi: 10.1007 / s00784-017-2063-9

8. Schär, M.O.; Diaz-Romero, J.; Kohl, S.; Zumstein, M.A.; Nesic, D.; Platelet-rich Concentrates Differentially Release Growth Factors and Induce Cell Migration In Vitro. Clin. Orthop. Relat. Res., 2015, 473, 1635-1643. https://doi: 10.1007/s11999-015-4192-2

9. Miron, R.J.; Chai, J.; Fujioka-Kobayashi, M.; Sculean, A.; Zhang, Y.; Evaluation of 24 protocols for the production of platelet-rich fibrin, in press, http:// doi:10.21203/rs.2.20730/v1

10. Crisci, A.; Lombardi, D.; Serra, E.; Lombardi, G.; Cardillo, F.; Crisci, M.; Standardized protocol proposed for clinical use of L-PRF and the use of L-PRF Wound Box ${ }^{\circledR}$. J. Unexplored Med. Data, 2017, $2,77-87$. http://doi:10.20517/2572-8180.2017.17.

11. Crisci, A.; Lombardi, D.; Serra, E.; Lombardi, G.; Cardillo, F.; Crisci, M.; L-PRF: standardized protocol proposed for the use of fibrin rich in leukocite platelet and the use of L-PRF Wound Box. Selection of an animal model. Update in Plastic Surgery, 2017, 3, 141-149.

12. Crisci, A.; The L-PRF Membrane (Fibrin Rich in Platelets and Leukocytes) and Its Derivatives (A-PRF, I-PRF) Are Useful as a Source of Stem Cells in Regenerative Wound Therapy: Experimental Work on the Horse. Regen. Med. Ther., 2019, 3, 37-45. http://doi: 10.36959/654/392

13. D’asta, F.; Halstead, F.; Harrison, P.; Zecchi Orlandini, S.; Moiemen, N.; Lord, J.; The contribution of leucocytes to the antimicrobial activity of platelet-rich plasma preparations: A systematic review, Platelets, 2018, 29, 9-20. http://doi:10.1080/09537104.2017.1317731

14. Kawase, T.; Mubarak, S.; Mourão, C.F.;The Platelet Concentrates Therapy: From the Biased Past to the Anticipated Future, Bioengineering, 2020, 7, 82. https://doi.org/10.3390/bioengineering7030082

15. Apostólico, J.S.; Lunardelli, V.A.; Coirada, F.C.; Boscardin, S.B.; Rosa, D.S.; Adjuvants: Classification, Modus Operandi, and Licensing., J. Immunol. Res., 2016, 1459394. https://doi.org/10.1155/2016/1459394

16. Crisci, A.; La gestione dell'osteomielite nel piede diabetico. In: Crisci A. Il piede diabetico: nuove prospettive di prevenzione e cure, Ed. Aracne, Roma; 2014, 109-113.

17. Madurantakam, P.; Yoganarasimha, S.; Hasan, F.K.; Characterization of leukocyte-platelet rich Fibrin, a novel biomaterial., J. Vis. Exp., 2015, 29, 53221. https://doi: 10.3791/53221

18. Cieslik-Bielecka, A.; Dohan Ehrenfest, D.M.; Lubkowska, A.; Bielecki, T.; Microbicidal properties of leukocyte-and plate- let-rich plasma/fibrin (L-PRP/L-PRF): new perspectives., J.B.R.H.A., 2012, 2, 43-52.

19. Crisci, A.; Benincasa, G.; Crisci, M.; Crisci, F., Leukocyte Platelet Rich Fibrin (L-PRF), a new bio membrane useful in tissue repair: basic science and literature review., Bio. Interface Res. Appl. Chem., 2018, 5, 3635-3643.

20. Crisci, A.; Crisci, F.; Crisci, M.; Second-Generation Platelet Concentrates (L-PRF, A-PRF, i-PRF, i-PRF $M$, i-PRF+) in Cutaneous Wound Surgery of the Foot., Adv. Res. Foot Ankle, 2019, 2, 111.

https://doi: 10.29011/ARFA-111.1000011

21. O'Connell, S.M.; Hessler, K.; Darik, H.; Cascade Autologus System pletelet-rich fibrin in the treatment of chronic leg ulcers. Advanced in wound care, 2012, 1, 52-55. https://doi: 10.1089/wound.2011.0290

22.Crisci, A.; D'Adamo, R.; Crisci, M.; The Second-Generation Platelet Concentrates in the Treatment of Chronic Osteomyelitis: one Modern Regenerative Surgery. International Journal of Research -GRANTHAALAYAH, 2020, 8(10), 112-122. https://doi.org/10.29121/granthaalayah.v8.i10.2020.1842 